ARTICLE

https://doi.org/10.1038/s41467-019-11769-7

\title{
GIGANTEA recruits the UBP12 and UBP13 deubiquitylases to regulate accumulation of the ZTL photoreceptor complex
}

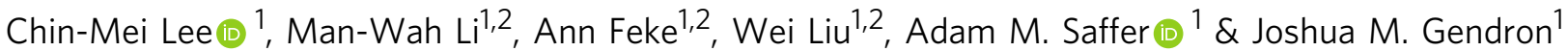

ZEITLUPE (ZTL), a photoreceptor with E3 ubiquitin ligase activity, communicates end-of-day light conditions to the plant circadian clock. It still remains unclear how ZTL protein accumulates in the light but does not destabilize target proteins before dusk. Two deubiquitylating enzymes, UBIQUITIN-SPECIFIC PROTEASE 12 and 13 (UBP12 and UBP13), which regulate clock period and protein ubiquitylation in a manner opposite to ZTL, associate with the ZTL protein complex. Here we demonstrate that the ZTL interacting partner, GIGANTEA (GI), recruits UBP12 and UBP13 to the ZTL photoreceptor complex. We show that loss of UBP12 and $U B P 13$ reduces $Z T L$ and $\mathrm{Gl}$ protein levels through a post-transcriptional mechanism. Furthermore, a ZTL target protein is unable to accumulate to normal levels in ubp mutants. This demonstrates that the ZTL photoreceptor complex contains both ubiquitin-conjugating and -deconjugating enzymes, and that these two opposing enzyme types are necessary for circadian clock pacing. This shows that deubiquitylating enzymes are a core element of circadian clocks, conserved from plants to animals.

\footnotetext{
${ }^{1}$ Department of Molecular, Cellular, and Developmental Biology, Yale University, New Haven, CT 06511, USA. ${ }^{2}$ These authors contributed equally: Man-Wah Li, Ann Feke, Wei Liu. Correspondence and requests for materials should be addressed to J.M.G. (email: joshua.gendron@yale.edu)
} 
C ircadian clocks in all organisms rely on photoreceptors to sense light and entrain the central oscillator. The exact timing of the light-to-dark transition (dusk) is especially important for plants, as this indicates the length of the day and provides seasonal timing information necessary for the adjustment of plant developmental processes ${ }^{1-8}$. One way that Arabidopsis senses the end of the day is by using a unique photoreceptor called ZEITLUPE (ZTL) to control the stability of circadian clock transcription factors differentially in the light and the dark ${ }^{9}$. ZTL contains an N-terminal light-oxygen-voltage sensing (LOV) domain, which senses blue light. Adjacent to the LOV domain are the F-box domain, which allows ZTL to function as an E3 ubiquitin ligase, and a Kelch-repeat domain. ZTL mediates degradation of transcription factors that are at the core of the plant circadian clock, including TIMING OF CAB2 EXPRESSION 1, PSEUDO-RESPONSE REGULATOR 5, and CCA1 HIKING EXPEDITION (TOC1, PRR5, and CHE) ${ }^{10-15}$. In the light, ZTL accumulates to high levels but is unable to mediate degradation of the clock transcription factors ${ }^{16,17}$. The accumulation of ZTL protein during the day is dependent on interaction with the co-chaperone protein GIGANTEA $(\mathrm{GI})^{18-20}$. GI interacts with ZTL through the LOV domain in the light and dissociates from ZTL in the dark, allowing ZTL to mediate degradation of its target proteins and then be degraded by the ubiquitin proteasome system, likely through autocatalytic activity $10,11,16-18,21,22$. One of the roles of GI is to recruit HSP70/ HSP90 for maturation of the ZTL protein in the light, but ZTL is unable to mediate ubiquitylation and degradation of target proteins until dark ${ }^{10-12,19,23}$. It was proposed that GI can promote maturation of ZTL and block or counteract ZTL activity; however, this second role for GI has not been investigated in depth 12,23 .

We previously identified ZTL-interacting proteins using immunoprecipitation followed by mass spectrometry (IP-MS) with a "decoy" ZTL that lacks E3 ubiquitin ligase activity and stably binds interacting proteins ${ }^{14}$. We identified UBIQUITINSPECIFIC PROTEASE 12 and 13 (UBP12 and UBP13) as highconfidence ZTL-interacting proteins, which were shown previously to have an unspecified role in clock function ${ }^{14,24}$. UBP12 and UBP13 also interact with GI in IP-MS experiments ${ }^{25}$, suggesting that either the UBPs interact with ZTL and GI independently or that ZTL, GI, and the UBPs exist together in a complex. UBP12 and UBP13 are closely related deubiquitylating enzymes that can cleave lysine 48-linked mono- or poly-ubiquitin from substrates ${ }^{24,26}$, interestingly, a biochemical role opposite to that of ZTL. In addition to regulating the circadian clock, they are also involved in flowering time, pathogen defense, root differentiation, and hormone signaling $26-30$.

Here, we show that UBP12 and UBP13 interact with the ZTL photoreceptor complex in a GI-dependent manner. Supporting this idea, genetic analyses show that UBP12 and UBP13 impact clock function through the same genetic pathway as ZTL and GI. Finally, we demonstrate that UBP12 and UBP13 are necessary for the proper daily accumulation of ZTL, GI, and TOC1 proteins. These results support the idea that in plants the communication of end-of-day light information relies on a photoreceptor complex that contains both ubiquitin conjugation activity and ubiquitin deconjugation activity.

\section{Results}

ZTL, GI, and UBP12/UBP13 form a trimeric complex. Previously, it was shown that UBP12 and UBP13 associate with ZTL and GI in vivo ${ }^{14,25}$. To test whether UBP12 or UBP13 proteins interact with the members of the ZTL/GI protein complex we performed yeast two-hybrid assays. We found that UBP12 and
UBP13 interacted with GI but not with ZTL or the ZTL target proteins TOC1, PRR5, or CHE (Fig. 1a). We next tested the interaction between GI and UBP12 and UBP13 in planta via bimolecular fluorescence complementation (BiFC) in Arabidopsis protoplasts (Fig. 1b). GI, UBP12, and UBP13 are localized in the cytoplasm and nucleus ${ }^{18,24}$, and our BiFC results show that UBP12 and UBP13 interact with GI in both compartments with strong signal in the nucleus and weaker but detectable signal in the cytoplasm. The interacting complexes of UBP12 and GI formed nuclear foci, similar to the localization of GI alone ${ }^{31}$. UBP12 and UBP13 contain a MATH-type (meprin and TRAF homology) protein interaction domain and a ubiquitin-specific protease (USP) domain (Supplementary Fig. 1). The MATH domains of UBP12 and UBP13 were necessary for interaction with GI while the protease domain and the C-terminal portions did not mediate GI-interaction (Fig. 1c). This suggests that the interaction between GI and UBP12 or UBP13 is not dependent on the UBP USP domains binding to poly-ubiquitylated GI protein.

We next determined whether GI was necessary to bridge the interaction between UBP12 or UBP13 and ZTL in vivo by performing IP-MS on wild-type (Col-0) and gi-2 mutant transgenic lines expressing the decoy ZTL protein (Supplementary Fig. 2). We collected samples at $9 \mathrm{~h}$ after dawn from plants grown in $12 \mathrm{~h}$ light $/ 12 \mathrm{~h}$ dark cycles to capture the time when ZTL and GI are normally interacting. We found that UBP12 and UBP13 were enriched in the Col-0 samples ( $p$-value $=3.58 \mathrm{E}-5$ and 0.0113 for UBP12 and UBP13, respectively), but not in the gi2 mutant $(p$-value $=1$ for both) (Fig. $1 \mathrm{~d}$ and Supplementary Data 1). These results indicate that GI is required for UBP12/ UBP13 to form a complex with ZTL, substantiating our interaction studies in heterologous systems. Notably, LKP2, a known ZTL-interacting partner, associated with ZTL in the presence or absence of GI and suggests that the decoy ZTL is able to form biologically relevant protein complexes even in the gi-2 mutant $^{32}$. Altogether these results suggest that the GI protein physically bridges the interaction between UBP12 or UBP13 and ZTL in vivo.

As a complementary approach to the IP-MS (Fig. 1d) we coexpressed FLAG-UBP12 or FLAG-UBP13 with HA-GI and MycZTL in N. benthamiana leaves. We then performed immunoprecipitation with anti-FLAG antibody and detected the presence of FLAG-UBP12, FLAG-UBP13, HA-GI, and Myc-ZTL using western blotting (Fig. 1e). In the FLAG immunoprecipitation samples, HA-GI was always detected when co-expressed with FLAG-UBP12 or FLAG-UBP13, showing that UBP12 and UBP13 interact with GI independently of the presence of Myc-ZTL. Furthermore, Myc-ZTL was undetectable in the FLAG immunoprecipitation samples unless co-expressed with HA-GI showing that the interaction between UBP12 or UBP13 and ZTL is dependent on GI. These assays support our previous results (Fig. 1a-d) and show that a trimeric complex between full-length ZTL, GI, and UBP12 or UBP13 can form in vivo (Fig. 1f).

$U B P 12 / U B P 13$ are in the same genetic pathway as $Z T L$ and GI. Our physical interaction model (Fig. 1f) led us to hypothesize that $U B P 12$ and UBP13 regulate the circadian clock through the same genetic pathway as ZTL and GI. We tested this via epistasis analyses with loss-of-function mutants in ZTL, GI, UBP12, and $U B P 13$. Previously, it was shown that knockdown of UBP12 and $U B P 13$ results in shortened clock periods ${ }^{24}$. We first determined the period of a series of mutant alleles in $U B P 12$ and $U B P 13$ by crossing them to the $p C C A 1:: L U C$ clock reporter transgenic line and measuring luciferase activity (Fig. $2 \mathrm{a}-\mathrm{d}$ ). We found that single mutations in either UBP12 or UBP13 shortened the clock 
a
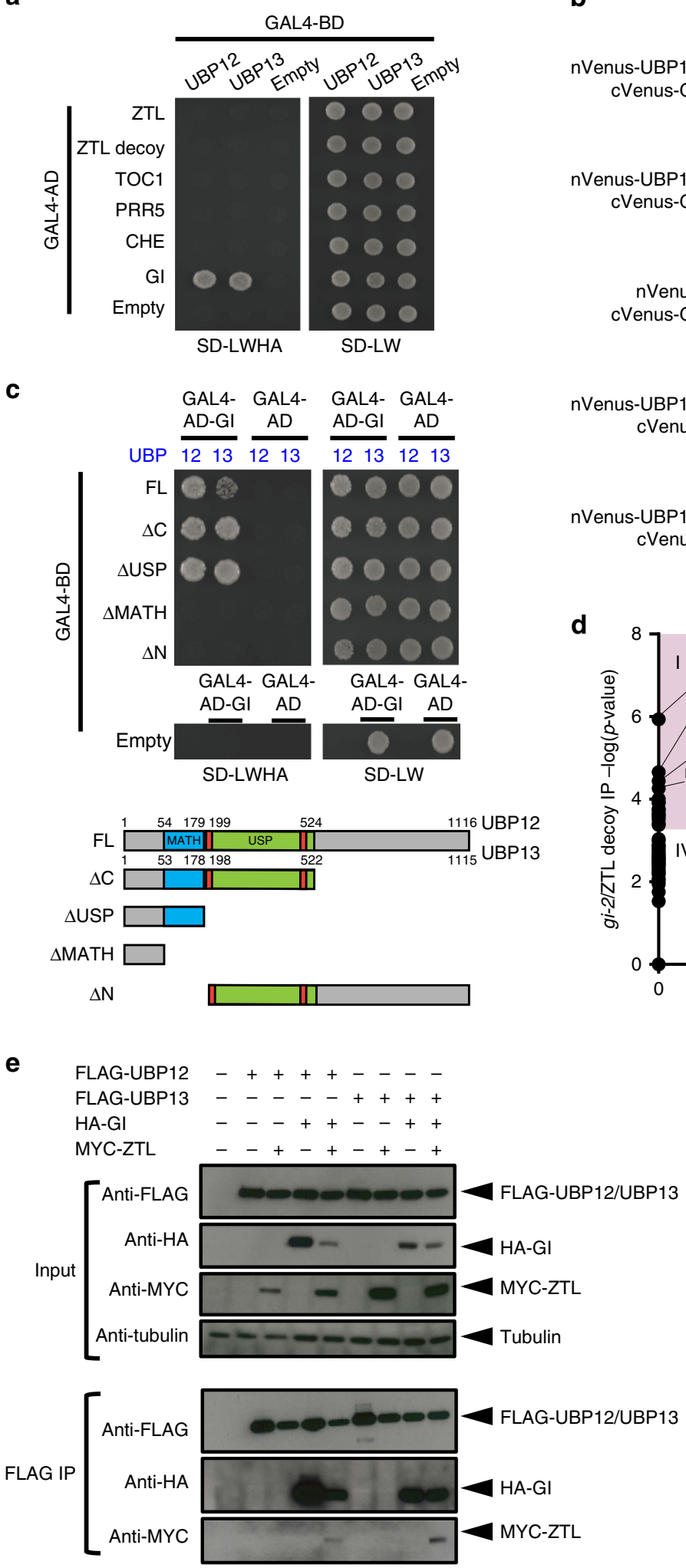

b

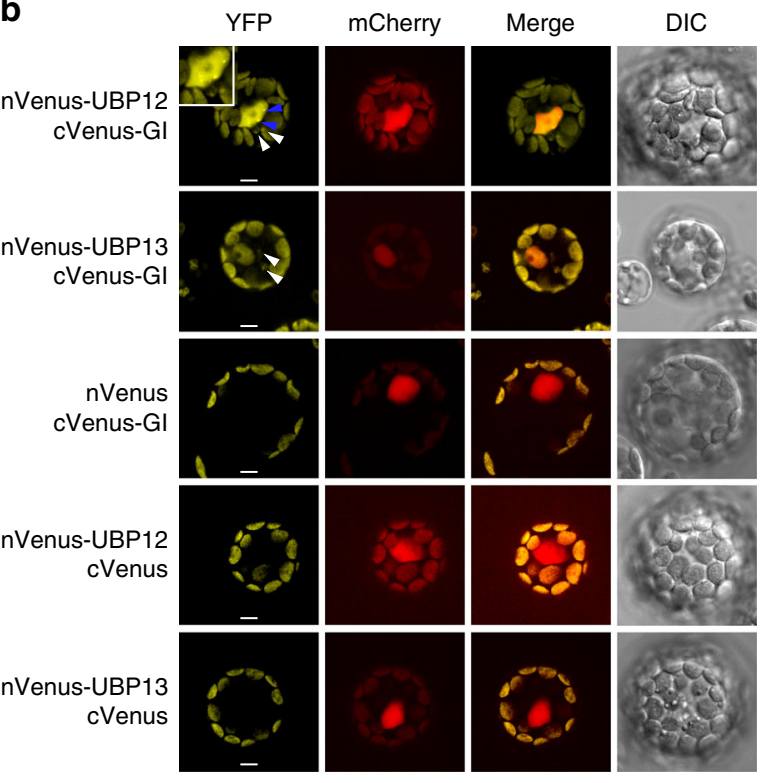

d

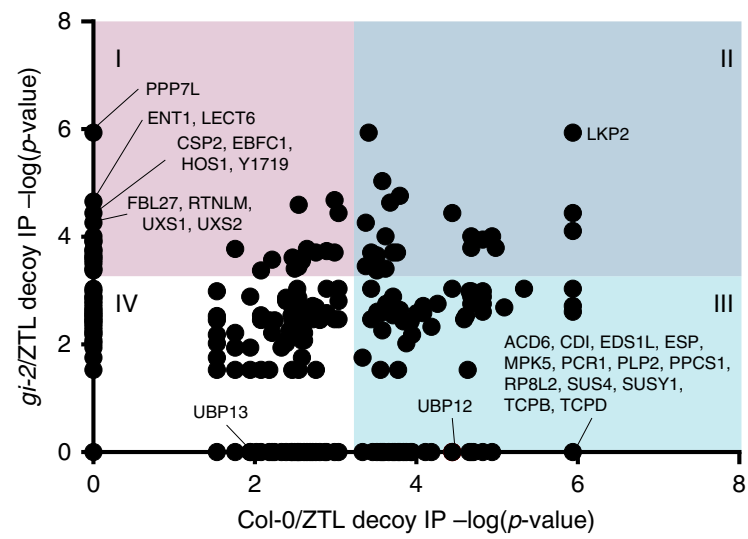

period with period lengths that varied from 0.4 to $1 \mathrm{~h}$ shorter than wild type. We next generated ubp12-1/gi-2 and ubp13-1/gi-2 double mutants and measured the expression of the core clock gene CCA1 during a 2-day circadian time course in constant light using quantitative reverse-transcription PCR (qRT-PCR) (Fig. 2e, $\mathrm{f}$ and Supplementary Table 1). LS Periodogram analysis using the Biodare2 platform [biodare2.ed.ac.uk ${ }^{33}$ ] showed that the $u b p 12-1 /$ gi-2 double mutant had a similar phase and amplitude of CCA1 expression to the gi-2 mutant alone and a period more similar to ubp12-1 (Supplementary Table 2). These results show a nonadditive interaction and suggest they function in the same circadian genetic pathway. The $u b p 13-1 / g i-2$ double mutant had a similar amplitude to the gi-2 mutant but had a more similar phase and period to the $u b p 13-1$ mutant (Supplementary Table 2). This 
Fig. $1 \mathrm{Gl}$ bridges the interactions between ZTL and UBP12 or UBP13. a Yeast two-hybrid showing interaction between GI and UBP12 or UBP13. The GAL4 DNA-binding domain (GAL4-BD) fused to UBP12 or UBP13 and either ZTL variants (ZTL and ZTL decoy), ZTL targets (TOC1, PRR5, and CHE) or GI fused to GAL4 activation domain (GAL4-AD) were grown on SD-LW medium for autotrophic selection and on SD-LWHA medium to test for interactions. b Bimolecular fluorescence complementation (BiFC) assays to examine the interactions of UBP12 or UBP13 and GI fused to the N-or C-terminus of Venus (YFP) were performed in Arabidopsis protoplasts. The blue arrows indicate the interacting complex forming nuclear foci. The white arrows show fluorescence signal in the cytoplasm. mCherry-VirD2NLS was co-expressed as a nuclear marker, and the scale bar indicates $10 \mu \mathrm{m}$. $\mathbf{c}$ The protein domains of UBP12 and UBP13 required to interact with GI were tested using yeast two-hybrid assays. The full-length (FL) or truncated UBP12 or UBP13 fragments as diagramed in the lower portion of the panel were fused to GAL4-BD to test for interaction with GAL4-AD-GI. d Scatter plot of proteins identified by IP-MS of ZTL decoys in the Col-0 and gi-2 genotypes. The significance of the interactions were evaluated by SAINTexpress (see Methods and Supplementary Data 1 for complete information) with a false discovery rate (FDR) cutoff $<0.01$ and $p$-value $\leq 5.37 \mathrm{E}-4$ to separate interacting proteins into four groups. Group I: significant interactions with ZTL decoy in the gi-2 but not Col-0. Group II: significant interactions with ZTL decoy in both Col-0 and gi-2. Group III: significant interactions with ZTL decoy in the Col-0 but not gi-2. Group IV: Non-significant interactions with ZTL decoy in both Col-0 and gi-2. The interacting proteins significantly enriched in the gi-2 mutant over $\mathrm{Col}-0$ were labeled along the $y$-axis, and the proteins enriched in the Col-0 over the gi-2 mutant were labeled along the $x$-axis. e Co-immunoprecipitation assays showing that UBP12 or UBP13 interact with ZTL in a GI-dependent manner. FLAGUBP12 or FLAG-UBP13 were co-infiltrated with HA-GI and Myc-ZTL in Nicotiana benthamiana leaves. Anti-FLAG antibody was used to immunoprecipitate FLAG-UBP12 or FLAG-UBP13. Western blotting with anti-FLAG, anti-HA, or anti-Myc was used to detect the presence of FLAG-UBP12, FLAG-UBP13, HA$\mathrm{Gl}$, or Myc-ZTL in the immunoprecipitated samples and inputs. $\mathbf{f}$ The diagram depicts the interaction between GI and the MATH domain of UBP12 or UBP13, and between GI and the LOV domain of ZTL. The source data are provided as a Source Data file. Blot images were cropped from their original size, which can be found in Source Data file

again shows a non-additive genetic interaction but also suggests that the roles of UBP12 and UBP13 have slightly diverged with respect to clock function. We also crossed the gi-2 mutant with the $u b p 12-2 w$ mutant, which had reduced expression of both $U B P 12$ and UBP13 and the shortest clock period of the tested $u b p$ mutant alleles (Supplementary Fig. 3 and Fig. 2a-d). The pattern of CCA1 expression in the $u b p 12-2 w / g i-2$ double mutant was nearly identical to the gi-2 mutant, further confirming that the effects of the UBPs and GI are not additive (Supplementary Table 2). These results indicate that UBP12 and UBP13 work in the same pathway as GI to control clock function.

ZTL functions downstream of GI to regulate the circadian clock $^{17}$. Thus, we hypothesized that ZTL would function downstream of $U B P 12$ and $U B P 13$ as well. To test the genetic interaction between $U B P 12$ or UBP13 and ZTL, we crossed $u b p 12-1$ and $u b p 13-1$ to the $z t l-4$ null mutant (Fig. $2 \mathrm{~g}, \mathrm{~h}$ ). The daily expression patterns of CCA1 in the ubp12-1/ztl-4 and ubp13-1/ztl-4 double mutants were nearly identical to the $z t l-4$ mutant alone in phase and amplitude (Supplementary Table 2). Interestingly, the period data showed that the $u b p 12-1 / z t l-4$ was more similar to $z t l-4$ than $u b p 12-1$, but the $u b p 13-1 / z t l-4$ is more similar to $u b p 13-1$. These data suggest that ZTL is epistatic to $U B P 12$ and $U B P 13$ but that UBP13 has diverged in function from $U B P 12$. It is important to note that the qRT-PCR data are below the suggested resolution for Biodare 2 analysis, which can result in inaccurate period calls (i.e., ubp13-1 period is estimated by Biodare 2 as the same period as wild type in this experiment). These results corroborate our physical interaction studies and suggest that UBP12 and UBP13 regulate the circadian clock upstream of $Z T L$.

UBP12 deubiquitylase activity is required for clock function. UBP12 and UBP13 are functional deubiquitylases that can cleave poly-ubiquitin from generic substrates 24,26 . We tested whether this deubiquitylation activity is necessary for their role in circadian clock function. To do this, we performed complementation studies with wild-type UBP12 and mutant UBP12C208S. UBP12 ${ }^{\mathrm{C} 208 \mathrm{~S}}$ has a mutation in the cysteine-box of the USP enzymatic core (Supplementary Fig. 1) that renders it non-functional as a deubiquitylase $24,27,28$. We transformed UBP12-YFP or $U B P 12^{C 208 S_{-} Y F P}$ driven by the UBP12 native promoter into the ubp12-1 mutant and analyzed a population of T1 transgenic lines. In this experiment we consider a line to have rescued the $u b p 12-1$ mutant clock phenotype if it has a period length longer than the average period length of the $u b p 12-1$ plus one standard deviation. Using this criteria, 10 of 32 transgenic lines (31\%) transformed with catalytically active UBP12 rescued the short period defect of the ubp12-1 mutant. Strikingly, only one transgenic line transformed with the inactive UBP12 ${ }^{\mathrm{C} 208 \mathrm{~S}}$ was able to rescue the short period phenotype of $u b p 12-1$ (Fig. $2 \mathrm{i}, \mathrm{j}$ ). As reference, $\sim 13 \%$ of the ubp12-1 plants themselves and $62 \%$ of the wild-type plants fell into the rescue category. This is likely due to normal variations in population level data of this type. We further confirmed that UBP12-YFP and UBP12 $208 \mathrm{~S}$-YFP were both localized to the cytoplasm and nucleus (Supplementary Fig. 4a), and that there is no observable effect of the C208S mutation on UBP12 stability (Supplementary Fig. 4b, c) when these proteins are transiently expressed. This suggests that differences between the wild type and C208S variants of UBP12 are not due to mislocalization or altered accumulation of the UBP12 $208 \mathrm{~S}$ protein. Altogether, these results indicate that the deubiquitylating functions of UBP12 are necessary for its role in regulating the circadian clock.

UBP12/UP13 stabilize GI, ZTL, and TOC1. By cleaving polyubiquitin from proteins, deubiquitylase enzymes can regulate protein stability and accumulation $28,30,34,35$. The physical and genetic interactions shown for UBP12, UBP13, GI and ZTL prompted us to hypothesize that the UBP12 and UBP13 regulate GI or ZTL protein levels, allowing for accumulation of the proteins in the end of the day. We measured the level of HA-tagged GI under the control of the GI native promoter ( $p G I:: G I-H A)$ in the $u b p 12-1$ and $u b p 13-1$ mutants during a $12 \mathrm{~h}$ light/12 $\mathrm{h}$ dark time course (Fig. 3a). GI protein levels were $\sim 50 \%$ lower in the $u b p 12-1$ and $u b p 13-1$. Messenger RNA (mRNA) expression of GI$H A$ was also $\sim 25 \%$ lower than wild type at the peak of GI mRNA expression, ZT8 (Fig. 3b). This suggests that GI protein accumulation is partially dependent on UBP12 and UBP13, but that altered transcription of GI could also have an effect on GI protein.

Next, we measured ZTL protein levels in the ubp12-1 and ubp13-1 mutants (Fig. 3c). ZTL protein levels were substantially decreased in the $u b p 12-1$ and $u b p 13-1$ mutants throughout the entire day/night cycle. Overexposure of the immunoblot showed that a small amount of ZTL protein can still accumulate in the $u b p$ mutants (Fig. 3c). The expression of ZTL mRNA was largely unaffected in these lines (Fig. 3d), suggesting that the decrease in ZTL protein levels was caused by a post transcriptional mechanism. This is similar to the post transcriptional control of ZTL reported in gi loss-of-function mutants ${ }^{17}$, and indicates 

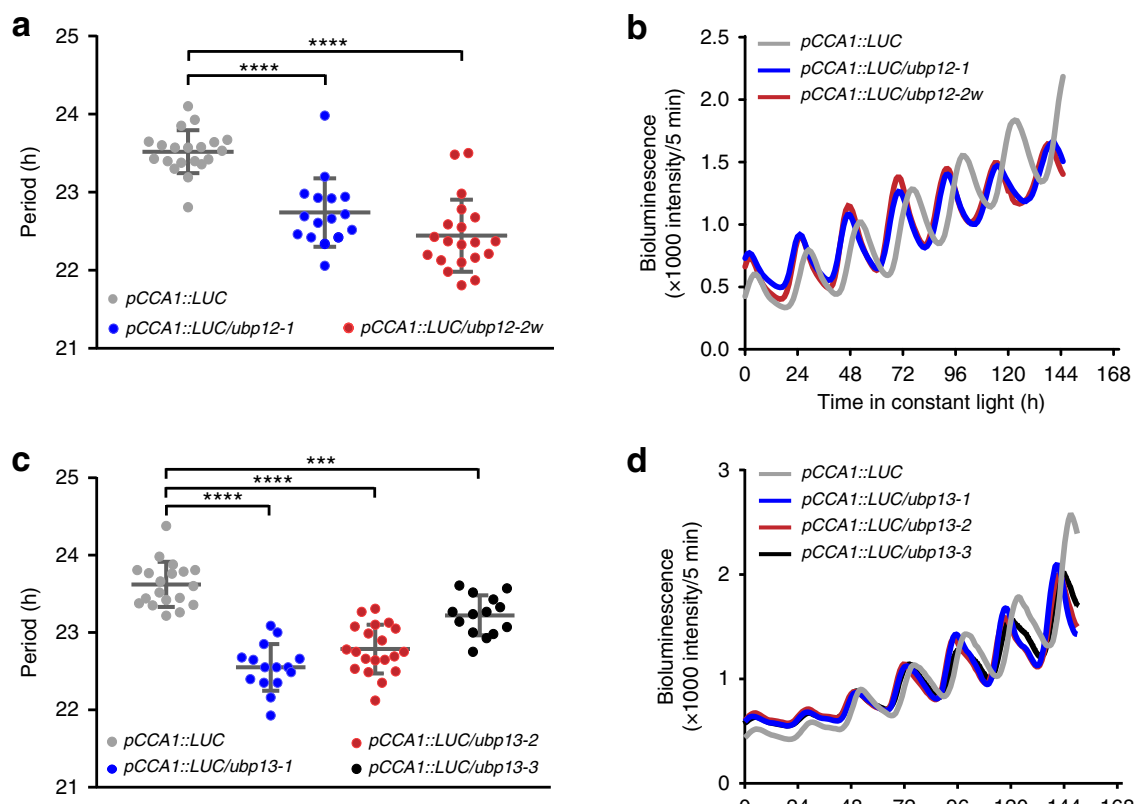

d
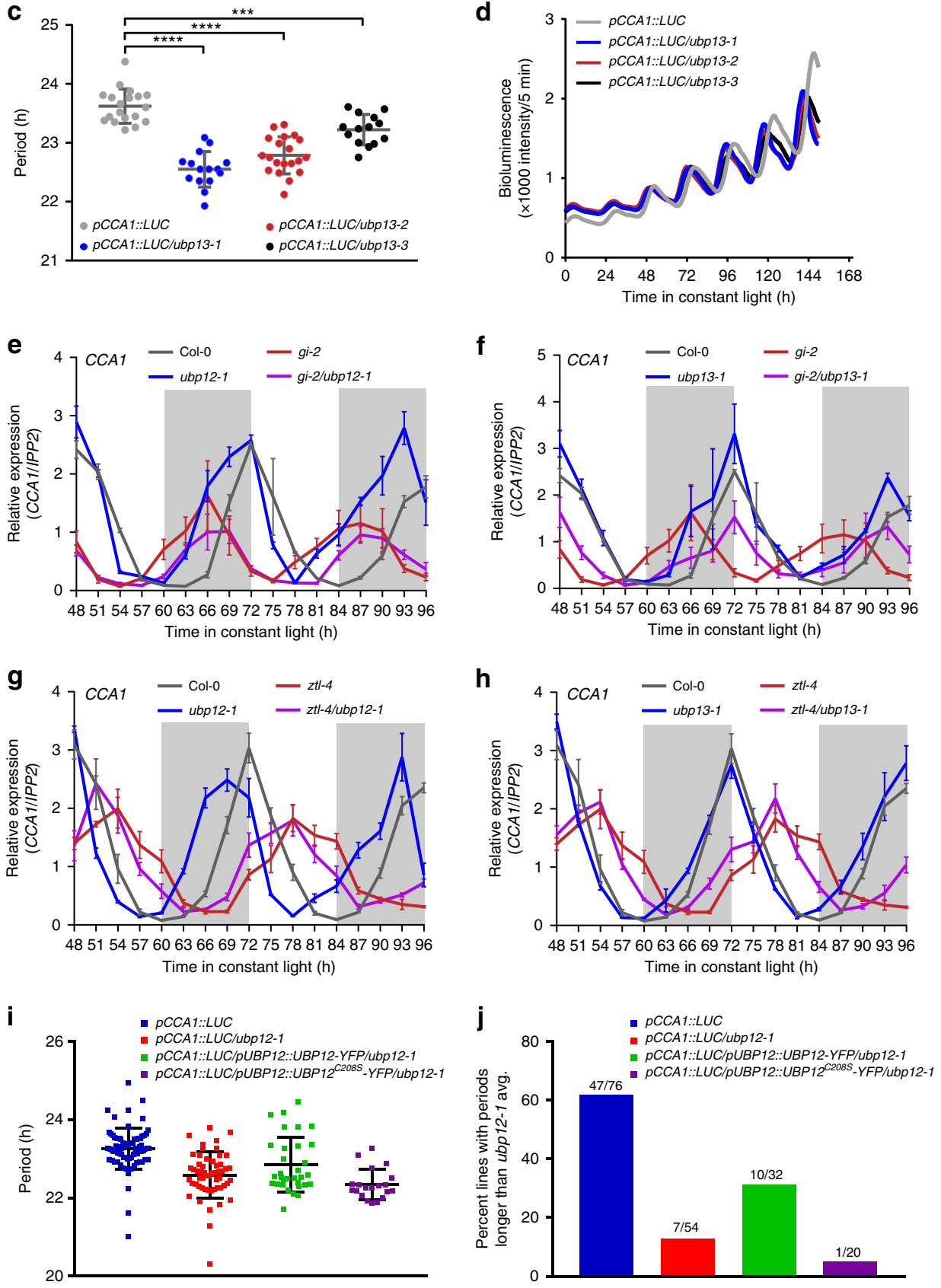

that UBP12 and UBP13 are necessary for robust accumulation of the ZTL protein.

Interestingly, the $u b p 12-1$ and $u b p 13-1$ mutants caused severe reduction in the levels of the ZTL protein but had a short period phenotype, opposite to the long period phenotype of $z t l$ loss-offunction mutants. Normally, loss of ZTL causes aberrantly high

levels of TOC1 protein while overexpression of ZTL causes low levels of TOC1 protein $10,11,23,36$. To determine if UBP12 and UBP13 affect TOC1 protein levels, we crossed a transgenic line expressing TOC1 fused to YFP under the TOC1 promoter $(T M G)$ to the $u b p 12-1$ and $u b p 13-1$ mutants and measured TOC1 protein levels (Fig. 3e). TOC1 protein levels were severely reduced in the 
Fig. 2 UBP12 and UBP13 regulate the circadian clock through the same pathway as GI and ZTL. a-d The ubp12 and ubp13 mutants have short period phenotypes. a, c The periods of circadian marker pCCA1:Luciferase ( $p$ CCA1::LUC) in the wild type (Col-0) $(n=20$ for $\mathbf{a}$ and $n=19$ for $\mathbf{c}$ ), ubp12-1 ( $n=16$ ), ubp12-2w $(n=20)$, ubp13-1 $(n=15)$, ubp13-2 $(n=20)$, and ubp13-3 $(n=14)$ were measured with bioluminescent assays. Each symbol represents the period from one seedling, and the average period and standard deviation are labeled with gray bars. The significance of period changes between wild type and mutants were analyzed with a two-tailed Welch's $t$-test ( ${ }^{\star \star \star}$ for $p$-value $<0.001$; ${ }^{\star \star \star \star}$ for $p$-value $<0.0001$ ). Three biological replicates were performed with similar results, and one dataset is presented. $\mathbf{b}, \mathbf{d}$ The average bioluminescence of the lines displayed in a and $\mathbf{c}$ were plotted against time after transfer from $12 \mathrm{~h}$ light/12 $\mathrm{h}$ dark entrainment conditions to constant light. e, f Circadian expression of CCA1 in Col-0, ubp12-1, ubp13-1, gi-2, gi-2/ubp12-1, and gi-2/ ubp13-1 after transferring to constant light for $48 \mathrm{~h}$ from the entrainment conditions was measured using qRT-PCR. Subjective dark is colored with light gray. The data represent the average relative expression of CCA1 normalized to IPP2 from three biological replicates, and the error bars are the standard deviation. The same Col-0 and gi-2 data were plotted twice (in $\mathbf{e}$ and $\mathbf{f}$ ) for clarity in the data presentation and for comparison with the other mutant lines. $\mathbf{g}$, $\mathbf{h}$ The circadian expression of CCA1 in Col-0, ubp12-1, ubp13-1, ztl-4, ztl-4/ubp12-1, and ztl-4/ubp13-1 after transferring to constant light for 48 $\mathrm{h}$ from the entrainment conditions was measured using qRT-PCR. The data analyses and presentation are the same as e-f. The same Col-0 and $z t /-4$ data were plotted twice (in $\mathbf{g}$ and $\mathbf{h}$ ). $\mathbf{i}$ The circadian period of $p C C A 1:: L U C$ in the wild type $(n=76)$, ubp12-1 $(n=54)$, ubp12-1 mutant complemented with $p U B P 12:: U B P 12-Y F P$ $(n=32)$ or deubiquitylating activity-dead pUBP12::UBP12CS-YFP $(n=20)$. Each symbol represents the period from one seedling, and the black bars indicate the average period and standard deviation. The wild type and ubp12-1 mutants are homogenous populations, and the complementation lines are individual $\mathrm{T} 1$ transgenic lines. The presented data are from three independent biological replicates. $\mathbf{j}$ Quantitation of the number of lines, from panel $\mathbf{i}$, with periods greater than the average of the ubp12-1 mutant plus one standard deviation. The source data are provided as a Source Data file

$u b p 12-1$ and $u b p 13-1$ mutants while mRNA expression of the TOC1-YFP transgene was similar in the wild type and mutant backgrounds, suggesting that the decrease in TOC1 protein levels was caused by a post-transcriptional mechanism (Fig. 3f). Notably, TOC1 protein was unable to accumulate to high levels in the light in the $u b p$ mutants (Fig. $3 \mathrm{e}$ at $12 \mathrm{~h}$ after dawn). This is similar to the effects of the gi-2 mutant, where TOC1 protein levels never accumulate to full wild-type levels ${ }^{17}$. This suggests that the period effects of the $u b p 12$ and $u b p 13$ mutants may be caused by the same mechanism as the short period of the gi-2 mutant.

\section{Discussion}

We have shown that UBP12 and UBP13 are components of the ZTL-GI photoreceptor complex that are necessary for accumulation of the proteins in the end of the day. UBP12 and UBP13 can remove poly-ubiquitin from targets non-specifically ${ }^{24,26}$. Thus, we hypothesize that UBP enzymes are recruited by GI to the ZTL photoreceptor complex to prevent formation of polyubiquitin chains, resulting in increased stability of the protein complex (Fig. 4). Interestingly, ZTL protein levels were severely damped in the $u b p 12$ and $u b p 13$ mutants, but counterintuitively the ZTL target, TOC1, also had reduced levels (Fig. 3c-f). This effect is similar to what was observed in a gi loss-of-function mutant, and suggests that GI and UBP12 and UBP13 can counterbalance the activity of ZTL during the day, allowing TOC1 to accumulate to high levels before being degraded ${ }^{17}$. Although ZTL levels were decreased in the $u b p$ mutants, there was still a small amount that could potentially decrease TOC1 levels in the light (Fig. 3c long exposure). This is different than what was seen when HSP90 activity was inhibited, resulting in lower ZTL levels but higher TOC1 levels. This suggests that HSP90 is necessary for ZTL protein maturation and to promote its activity ${ }^{22}$. These data in combination with our results suggest that GI performs two roles in the ZTL photoreceptor complex: (1) acting as a co-chaperone that recruits HSP proteins to facilitate ZTL maturation ${ }^{19,20}$, and (2) counterbalancing the role of ZTL in ubiquitin conjugation with UBP12 and UBP13 present to deconjugate ubiquitin. The light-regulated nature of the ZTL-GI interaction also indicates that light is controlling the balance of ubiquitin conjugation and deconjugation that allows the ZTL photoreceptor complex to accurately degrade proteins at the correct time of day. It was previously shown that mammalian and insect circadian clocks utilize deubiquitylation to regulate stability and subcellular localization of clock proteins ${ }^{37-39}$. In light of this, our results further demonstrate that deubiquitylation activity is an evolutionarily conserved feature of the clocks of higher eukaryotes Furthermore, the mammalian ortholog of UBP12 and UBP13, USP7, impacts clock function in response to environmental stress ${ }^{40,41}$, suggesting that these deubiquitylases are conserved clock regulators across evolution.

\section{Methods}

Plant materials and growth conditions. The Arabidopsis seeds of Col-0, ubp12-1 (CS423387), ubp12-2w (CS2103163), ubp13-1 (SALK 128312), ubp13-2 (SALK_024054), ubp13-3 (SALK_132368) $)^{24}, g i-2(\operatorname{cs} 3370)^{42,43}, z t l-4$ $\left(\right.$ SALK_012440) $^{44}$, p GI::GI-HA (CS66130) ${ }^{45}$, and TMG $(\mathrm{CS} 31390)^{10}$ were described previously and obtained from ABRC. The ubp12-1/gi-2, ubp12-2w/gi-2, ubp13$1 / g i-2, u b p 12-1 / z t l-4$, and $u b p 13-1 / z t l-4$ double mutants were generated by crossing and genotyped by PCR. The pGI::GI-HA and TMG lines were crossed to ubp12-1 and $u b p 13-1$, and the homozygous lines were selected by genotyping and gentamycin resistance.

For IP-MS, the 35S::FLAG-His-ZTL-decoy transgenic lines and 35S::FLAG-HisGFP control were described previously ${ }^{46}$, and the same constructs were transformed into the $g i$-2 background by floral-dip method ${ }^{47}$

For the bioluminescent assays, the circadian reporter line pCCA1::Luciferase ( $p C C A 1:: L U C)^{48}$ was crossed to the $u b p 12$ and $u b p 13$ mutants. The $p U B P 12::$ UBP12-YFP variants (see Cloning section) were transformed into $p C C A 1:: L U C /$ ubp12-1 by floral-dip ${ }^{47}$ for complementation experiments.

For growth conditions of Arabidopsis seedlings, the seeds were surface sterilized with ethanol, cold stratified, plated on $1 / 2$ strength MS (Murashige and Skoog medium, Caisson Laboratories, MSP01) medium with $0.8 \%$ Agar (AmericanBio, $\mathrm{AB} 01185$ ), and grown at $22^{\circ} \mathrm{C}$ under $12 \mathrm{~h}$ light $/ 12 \mathrm{~h}$ dark as described previously ${ }^{46}$ unless specified otherwise. For soil-grown conditions, plants were grown in Fafard2 mix under $16 \mathrm{~h}$ light $/ 8 \mathrm{~h}$ dark at $22^{\circ} \mathrm{C}$.

For circadian experiments, seedlings were grown on $1 / 2$ strength MS medium under $12 \mathrm{~h}$ light $/ 12 \mathrm{~h}$ dark at $22^{\circ} \mathrm{C}$ for 10 days, transferred to continuous light (LL) at $22^{\circ} \mathrm{C}$ for $48 \mathrm{~h}$ before starting harvest. For the $12 \mathrm{~h}$ light $/ 12$ dark (LD) experiments, 12 -day-old seedlings grown on $1 / 2$ strength MS medium were used.

Cloning. The GATEWAY pENTR ${ }^{\mathrm{m} x} / \mathrm{D}-\mathrm{TOPO}$ entry vectors (Thermo Fisher Scientific, K240020) of ZTL full-length, ZTL decoy, CHE, TOC1, and PRR5 were obtained from previous reports ${ }^{46,48,49}$. For GI, UBP12 and UBP13, the full-length coding regions were amplified from complementary DNA by PCR and cloned into $\mathrm{pENTR}^{\mathrm{rm}} / \mathrm{D}$-TOPO vectors. These entry clones were then sub-cloned into GATEWAY compatible yeast two-hybrid vectors (pGADT7-GW and pGBKT7-GW) ${ }^{50}$ or BiFC vectors (pUC-DEST-VYCE ${ }^{\circledR} \mathrm{GW}$ and pUC-DEST-VYNE ${ }^{\circledR} \mathrm{GW}$ ) ${ }^{51}$ with GATEWAY recombination cloning (Thermo Fisher Scientific).

To construct the fragments of UBP12 and UBP13 into yeast two-hybrid pGADT7-GW vectors, the desired fragments were first amplified from the fulllength UBP12 or UBP13 entry vectors by PCR and cloned into $\mathrm{PENTR}{ }^{\mathrm{rx}} / \mathrm{D}-\mathrm{TOPO}$ vectors before being sub-cloned into pGADT7-GW with GATEWAY cloning.

For the UBP12 complementation plasmids, the pENTR ${ }^{\mathrm{sm}} / \mathrm{D}$-TOPO-UBP12-NS vector served as template for site-directed mutagenesis to introduce a Cys to Ser mutation at a.a. 208 position using Q5 ${ }^{\circledR}$ Site-Directed Mutagenesis Kit (NEB, E0554). Subsequently, UBP12-NS and UBP12C208S-NS in the $\mathrm{pENTR}^{\mathrm{ru}} / \mathrm{D}-\mathrm{TOPO}$ entry vectors were sub-cloned into a modified GATEWAY compatible pGreenBarT vector $^{51}$ with $1.7 \mathrm{k}$ bp upstream of ATG of UBP12 promoter region in the KpnI/ XhoI sites. The primers used for cloning were listed in Supplementary Table 1. 
a

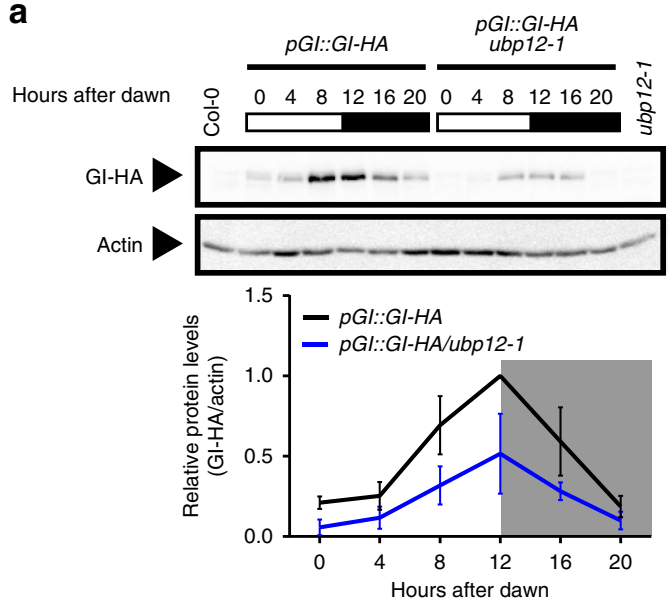

C
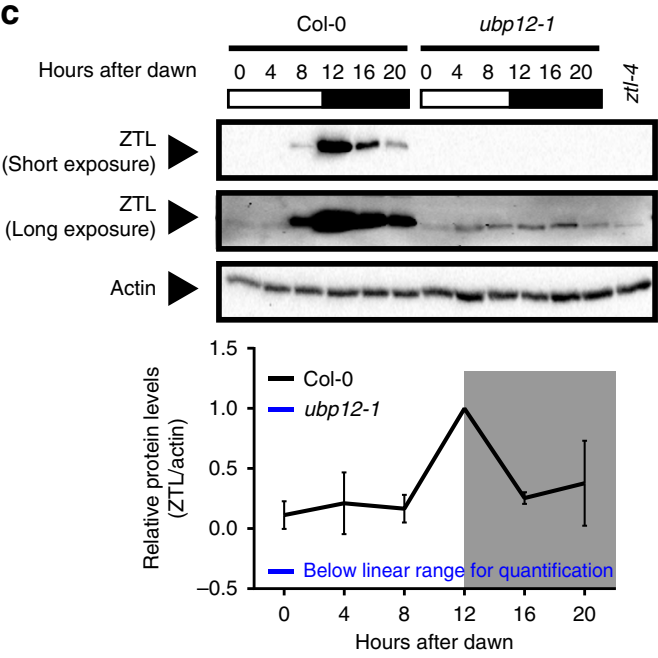

e

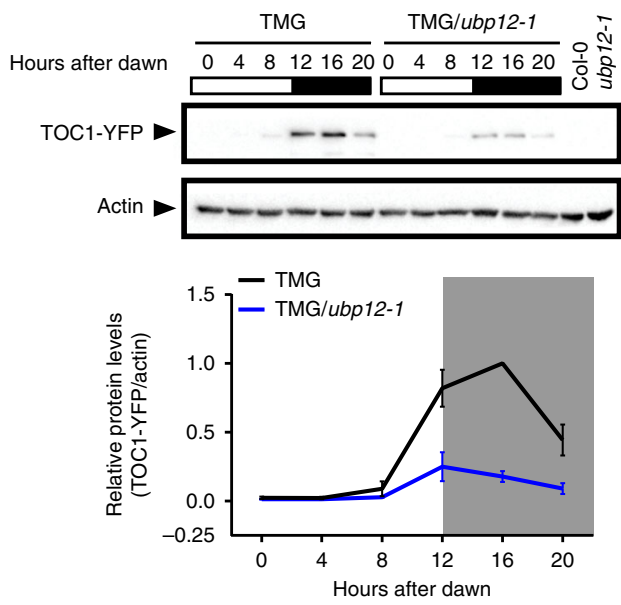

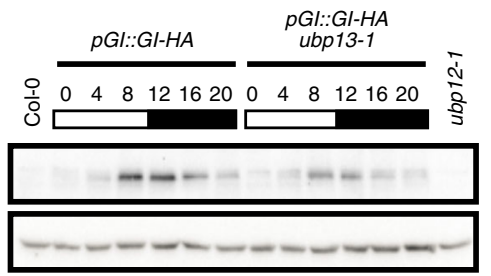
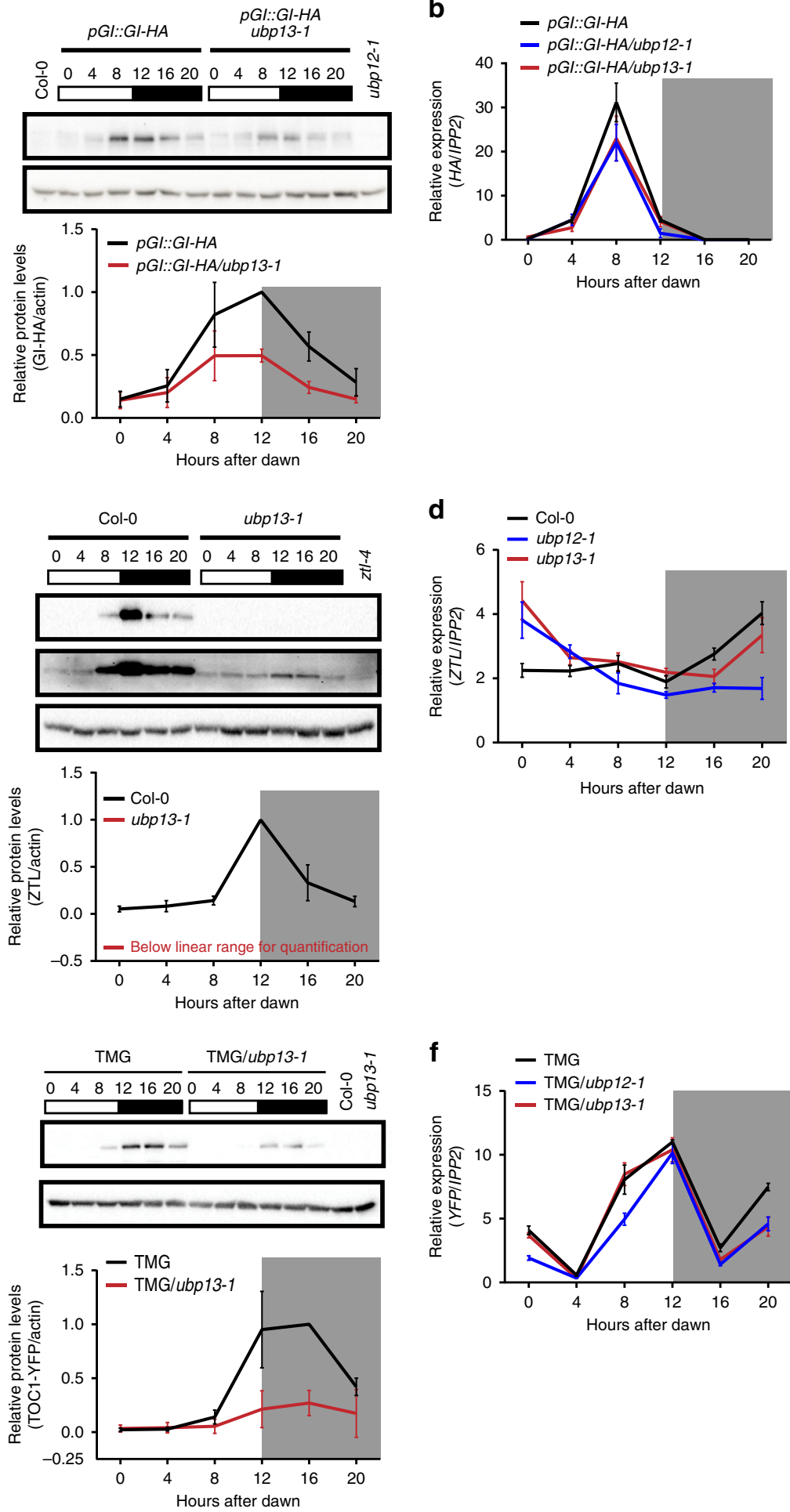

b

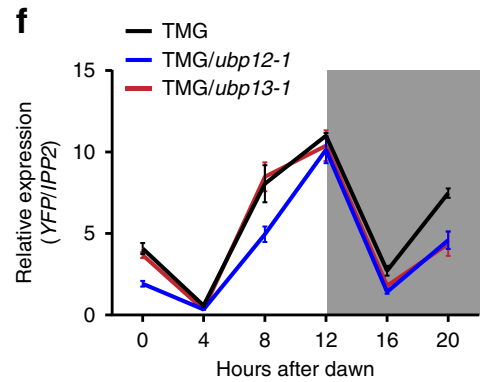

Fig. 3 ZTL, GI, and TOC1 protein levels are regulated by UBP12 and UBP13. a, c, e The protein levels of HA-tagged GI driven by native promoter ( $p$ Gl::GI$H A$ ), ZTL and YFP-tagged TOC1 driven by the TOC1 promoter (TOC1 minigene or TMG) in the wild type (Col-0), ubp12-1, or ubp13-1 mutants under diurnal conditions ( $12 \mathrm{~h}$ light/12 $\mathrm{h}$ dark) were detected by immunoblotting. The samples from $0 \mathrm{~h}$ to $12 \mathrm{~h}$ after dawn were harvested in light, and the samples from $16 \mathrm{~h}$ and $20 \mathrm{~h}$ after dawn were harvested in the dark (indicated by gray shading). The relative protein levels were quantified by normalization to actin. The Col-0 or ztl-4 samples were used as negative controls for the antibodies. Plots represent the average protein levels from three biological replicates, and the error bars represent standard deviation. Compared to wild type, the levels of ZTL proteins in the ubp12-1 and ubp13-1 were below the linear range for quantification. In the $z t /-4$ sample, the anti-ZTL antibody recognizes a non-specific band close to the size of endogenous ZTL in the long-exposure blots. b, $\mathbf{d}$, $\mathbf{f}$ The relative mRNA levels of GI-HA, ZTL, or TOC1-YFP from the same time course samples were measured by qRT-PCR. The source data are provided as a Source Data file. Blot images were cropped from their original size, which can be found in Source Data file 

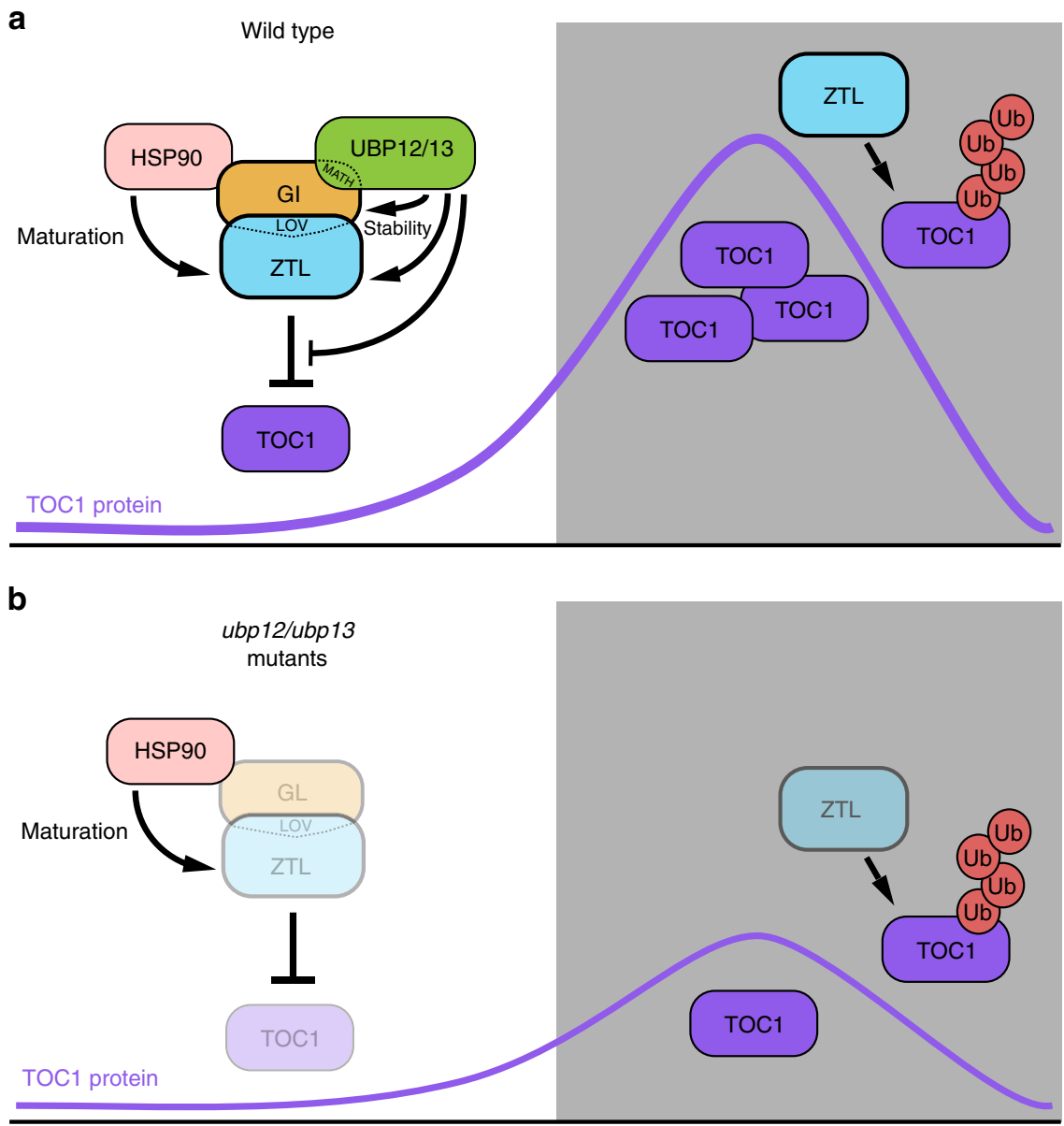

Fig. 4 The proposed model for UBP12/UBP13 regulation of ZTL. a In the light, GI interacts with ZTL and acts as a co-chaperone, recruiting HSP90 to facilitate folding and maturation of the ZTL protein. Additionally, Gl physically bridges an interaction between ZTL and UBP12 or UBP13. UBP12 or UBP13 stabilize the GI-ZTL protein complex before dusk. In the dark, GI dissociates from ZTL, and ZTL mediates ubiquitylation and degradation of the TOC1 protein. b Loss of UBP12 or UBP13 causes instability of ZTL and GI. Interestingly, the TOC1 protein levels are also reduced by loss of UBP12 or UBP13, mimicking the gi loss-of-function mutant

Yeast two-hybrid. ZTL, ZTL decoy, GI, TOC1, PRR5 and CHE were fused to the GAL4-BD in pGBKT7-GW vectors, and the full-length or fragments of UBP12 and UBP13 were fused to the GAL4-AD in pGADT7-GW vectors by GATEWAY cloning. The interactions were tested on synthetic dropout medium as described previously ${ }^{46}$.

BiFC and confocal microscopy. The coding region of GI, UBP12, or UBP13 in the GATEWAY entry vectors were cloned into protoplast GATEWAY destination vectors pUC-DEST-VYCE ${ }^{\oplus} \mathrm{GW}$ and pUC-DEST-VYNE(R)GW ${ }^{51}$, respectively, for transient transfections into protoplasts. pSAT6-mCherry-VirD2NLS was used as a nuclear marker. The protoplasts were isolated from 3- to 4-week-old Arabidopsis $(\mathrm{Col}-0)$ grown at $22^{\circ} \mathrm{C}$ under $8 \mathrm{~h}$ light $/ 16 \mathrm{~h}$ dark and transfected following the protocol of tape-Arabidopsis sandwich method ${ }^{52}$. After $14-18 \mathrm{~h}$ incubation in lowlight conditions, protoplasts were imaged on a Nikon Ti microscope with using a $60 \times 1.4$ NA plan Apo objective lens as described previously ${ }^{53}$. The images were analyzed with FIJI ${ }^{54}$.

Immunoprecipitation and mass spectrometry (IP-MS). For the ZTL decoys in Col-0 background, homozygous 35S::FLAG-His-ZTL-decoy transgenic lines along with Col-0 and 35S::FLAG-His-GFP controls were used. For the ZTL decoys in the gi-2 background, three independent T2 transgenic lines of 35S::FLAG-His-ZTLdecoy/gi-2 and 35S::FLAG-His-GFP/gi-2 were selected on $1 / 2$ strength MS plates with $15 \mu \mathrm{g} / \mathrm{ml}$ ammonium glufosinate before being transferred to soil. Twenty-one-dayold soil-grown plants were entrained in $12 \mathrm{~h}$ light $/ 12 \mathrm{~h}$ dark at $22^{\circ} \mathrm{C}$ for 7 days prior to harvest. Leaf tissues were collected at $9 \mathrm{~h}$ after dawn for subsequent IP-MS. One-step IP-MS and MS spectral analyses were carried out as documented ${ }^{46}$ with minor changes. The MS/MS spectral were searched against the SwissProt_2017 tax: Arabidopsis thaliana (thale cress) database (February 2017) using MASCOT MS/ MS ion search engine version 2.6.055 with the following parameters: up to two missed cleavages; variable modifications included Acetyl (K), GlyGly(K), Oxidation (M), Phospho (ST), Phospho (Y); peptide tolerance $\pm 10 \mathrm{ppm} ; \mathrm{MS} / \mathrm{MS}$ tolerance \pm
5 Da; peptide charge $2+$ and $3+$. The protein lists identified by MASCOT were first filtered out non-specific interactions by removing proteins only present in the controls (Col-0, gi-2, 35S::FLAG-His-GFP/Col-0 and 35S::FLAG-His-GFP/gi-2). The SAINTexpress algorithm ${ }^{56,57}$ were further performed to determine the significance of protein-protein interactions.

Bioluminescent assays. The Arabidopsis seedlings bearing $p C C A 1:: L U C$ in the wild type (Col-0), $u b p 12$, or $u b p 13$ mutants were grown in $1 / 2$ strength MS medium and entrained in $12 \mathrm{~h}$ light $/ 12 \mathrm{~h}$ dark for 7 days prior to being transferred to new $1 / 2$ strength MS plates and constant light (LL) for circadian free-run experiments. For the various $P U B P 12:: U B P 12-Y F P$ complementation T1 lines in the pCCA1::LUC/ ubp 12-1 background, seedlings were first screened and entrained on the $1 / 2$ strength MS plates containing $7.5 \mu \mathrm{g} / \mathrm{ml}$ ammonium glufosinate prior to being transferred to $1 / 2$ strength MS medium and LL. The measurement of luciferase activities and analyses were described as previously ${ }^{46}$.

Real-time quantitative reverse-transcription PCR (qRT-PCR). RNA extraction, reverse-transcription, and constitution of $\mathrm{qPCR}$ reactions were followed as described previously ${ }^{46}$, except for minor modifications. Four-hundred nanograms of total RNA were used for reverse-transcription reactions. For semi-quantification of gene expression, IPP2 (AT3G02780) was used as an internal control. The relative expression represents means of $2^{(-\Delta \mathrm{CT})}$ from three biological replicates, in which $\triangle \mathrm{CT}=(\mathrm{CT}$ of Gene of Interest $-\mathrm{CT}$ of IPP2). The primers were listed in Supplementary Table 1.

Immunoblotting. The procedure of protein extraction from Arabidopsis seedlings, separation, detection with antibodies, and quantification are described as previously ${ }^{46}$, except $60 \mu \mathrm{g}$ total protein were used for immunoblotting. The primary antibodies used for detection are: for GI-HA, anti-HA-Biotin antibody (1:1000, 12158167001, Millipore-Sigma); for ZTL, anti-ZTL antibody ${ }^{16}$ (1:200); for TMG, 
anti-GFP (1:10000, ab-290, Abcam); for FLAG-ZTL decoy, anti-FLAG antibody (1:1000, F7425, Millipore-Sigma). To quantify expression levels, the levels of target proteins were normalized to actin (anti-Actin antibody, 1:2000, SAB4301137, Millipore-Sigma).

Transient expression and confocal microscopy. UBP12-NS and UBP12C208SNS in the $\mathrm{pENTR}^{\mathrm{N}} / \mathrm{D}-\mathrm{TOPO}$ vectors were sub-cloned into inducible GATEWAY destination pABindGFP vectors ${ }^{58}$ and transformed into the Agrobacterium tumefaciens strain GV3101 for transient expression in Nicotiana benthamiana. The Agrobacterium culture of pABindGFP-UBP12 or pABindGFP-UBP12C208S and the nuclear marker pABindcherry-AS2 ${ }^{59}$ were pelleted and resuspended in the infiltration solution (5\% (w/v) Sucrose, $450 \mu \mathrm{M}$ acetosyringone and $0.01 \%(\mathrm{v} / \mathrm{v})$ Silwet). The bacterial infiltration solution was incubated at $4{ }^{\circ} \mathrm{C}$ for $2 \mathrm{~h}$ before infiltrated into 5-week-old Nicotiana benthamiana leaves. After $20 \mathrm{~h}$ of infiltration, the protein expression was induced by spraying leaves with $20 \mu \mathrm{M} \beta$-estradiol in $0.1 \%$ Tween 20 . The leaves were imaged after $18 \mathrm{~h}$ of induction.

The leaf samples were imaged on a Zeiss LSM510 confocal microscope with a Plan-Apochromat $40 \times / 1.3$ Oil objective. GFP was excited using $488 \mathrm{~nm}$ Argon laser and observed through a 505/530 nm bandpass filter. mCherry was excited using $543 \mathrm{~nm}$ HeNe laser and observed through a 585/615 nm bandpass filter. The images were processed with $\mathrm{FIJI}^{54}$

co-IP in Nicotiana benthamiana. The full-length coding sequences of ZTL, GI, UBP12, and UBP13 in the pENTR ${ }^{\mathrm{m}} / \mathrm{D}-\mathrm{TOPO}$ vectors were sub-cloned into pEarlygate203, pEarlygate201 and pEarlygate 202 plant binary vectors ${ }^{60}$, respectively, and transformed into Agrobacterium tumefaciens strain GV3101. Agroinfiltration into Nicotiana benthamiana leaves was described in the previous section. In this co-immunoprecipitation experiment co-infiltration with P19 in the EHA105 Agrobacterium strain was used to increase expression of the transgenes. The leaf samples were harvested after $48 \mathrm{~h}$ of infiltration and snap frozen with liquid nitrogen. Protein extraction and co-immunoprecipitation with Anti-FLAG ${ }^{\oplus}$ M2 Magnetic Beads (M8823, Millipore-Sigma), and a one-step IP protocol was used as described previously ${ }^{46,61}$. The inputs and IP samples were resolved on NuPAGE 4-12\% Bis-Tris Protein Gels (NP0321, Thermo Fisher Scientific) for immunoblotting. The primary antibodies used for detection are: for MYC-ZTL, anti-MYC antibody (1:10000, C3956, Millipore-Sigma); for HA-GI, anti-HA antibody (1:5000, H3663, Millipore-Sigma); for FLAG-UBP12 and FLAG-UBP13, anti-FLAG antibody (1:5000, F1804, Millipore-Sigma); for loading control, antitubulin antibody (1:5000, T5168, Millipore-Sigma).

Reporting summary. Further information on research design is available in the Nature Research Reporting Summary linked to this article.

\section{Data availability}

The mass spectrometry proteomics data was deposited to the ProteomeXchange Consortium via the PRIDE partner repository ${ }^{62}$ (https://www.ebi.ac.uk/pride/archive/). It is accessible via identifier PXD014636. The source data for Figs. 1, 2, and 3 and Supplementary Figs. 3 and 4 are in the Source Data file. Additional data and materials reported in this study are available from the corresponding author upon request.

Received: 15 June 2018 Accepted: 29 July 2019

Published online: 21 August 2019

\section{References}

1. Ito, S., Song, Y. H. \& Imaizumi, T. LOV domain-containing F-box proteins: light-dependent protein degradation modules in Arabidopsis. Mol. Plant 5, 573-582 (2012).

2. Carre, I. A. Day-length perception and the photoperiodic regulation of flowering in Arabidopsis. J. Biol. Rhythms 16, 415-423 (2001).

3. Yanovsky, M. J. \& Kay, S. A. Molecular basis of seasonal time measurement in Arabidopsis. Nature 419, 308-312 (2002).

4. Imaizumi, T., Tran, H. G., Swartz, T. E., Briggs, W. R. \& Kay, S. A. FKF1 is essential for photoperiodic-specific light signalling in Arabidopsis. Nature 426, 302-306 (2003).

5. Salome, P. A. \& McClung, C. R. The Arabidopsis thaliana clock. J. Biol. Rhythms 19, 425-435 (2004).

6. Imaizumi, T. \& Kay, S. A. Photoperiodic control of flowering: not only by coincidence. Trends Plant Sci. 11, 550-558 (2006).

7. Nozue, K. et al. Rhythmic growth explained by coincidence between internal and external cues. Nature 448, 358-361 (2007).

8. Mizoguchi, T. \& Yoshida, R. Punctual coordination: switching on and off for flowering during a day. Plant Signal. Behav. 4, 113-115 (2009).

9. Somers, D. E., Schultz, T. F., Milnamow, M. \& Kay, S. A. ZEITLUPE encodes a novel clock-associated PAS protein from Arabidopsis. Cell 101, 319-329 (2000).
10. Mas, P., Kim, W. Y., Somers, D. E. \& Kay, S. A. Targeted degradation of TOC1 by ZTL modulates circadian function in Arabidopsis thaliana. Nature 426, 567-570 (2003)

11. Kiba, T., Henriques, R., Sakakibara, H. \& Chua, N. H. Targeted degradation of PSEUDO-RESPONSE REGULATOR5 by an SCFZTL complex regulates clock function and photomorphogenesis in Arabidopsis thaliana. Plant Cell 19, 2516-2530 (2007)

12. Fujiwara, S. et al. Post-translational regulation of the Arabidopsis circadian clock through selective proteolysis and phosphorylation of pseudo-response regulator proteins. J. Biol. Chem. 283, 23073-23083 (2008).

13. Baudry, A. et al. F-box proteins FKF1 and LKP2 act in concert with ZEITLUPE to control Arabidopsis clock progression. Plant Cell 22, 606-622 (2010).

14. Lee, C. -M. et al. Decoys untangle complicated redundancy and reveal targets of circadian clock F-box proteins. Plant Physiol. 177, 1170-1186. https://doi. org/10.1104/pp.18.00331 (2018).

15. Han, L., Mason, M., Risseeuw, E. P., Crosby, W. L. \& Somers, D. E. Formation of an SCF(ZTL) complex is required for proper regulation of circadian timing. Plant J. 40, 291-301 (2004).

16. Kim, W. Y., Geng, R. \& Somers, D. E. Circadian phase-specific degradation of the F-box protein ZTL is mediated by the proteasome. Proc. Natl Acad. Sci. USA 100, 4933-4938 (2003).

17. Kim, W. Y. et al. ZEITLUPE is a circadian photoreceptor stabilized by GIGANTEA in blue light. Nature 449, 356-360 (2007).

18. Kim, J., Geng, R., Gallenstein, R. A. \& Somers, D. E. The F-box protein ZEITLUPE controls stability and nucleocytoplasmic partitioning of GIGANTEA. Development (Camb., Engl.) 140, 4060-4069 (2013).

19. Cha, J. Y. et al. GIGANTEA is a co-chaperone which facilitates maturation of ZEITLUPE in the Arabidopsis circadian clock. Nat. Commun. 8, 3 (2017).

20. Cha, J. Y., Khaleda, L., Park, H. J. \& Kim, W. Y. A chaperone surveillance system in plant circadian rhythms. BMB Rep. 50, 235-236 (2017).

21. Somers, D. E., Kim, W. Y. \& Geng, R. The F-box protein ZEITLUPE confers dosage-dependent control on the circadian clock, photomorphogenesis, and flowering time. Plant Cell 16, 769-782 (2004).

22. Kim, T. S. et al. HSP90 functions in the circadian clock through stabilization of the client F-box protein ZEITLUPE. Proc. Natl Acad. Sci. USA 108, 16843-16848 (2011).

23. Pudasaini, A. et al. Kinetics of the LOV domain of ZEITLUPE determine its circadian function in Arabidopsis. Elife 6, e21646 (2017).

24. Cui, X. et al. Ubiquitin-specific proteases UBP12 and UBP13 act in circadian clock and photoperiodic flowering regulation in Arabidopsis. Plant Physiol. 162, 897-906 (2013).

25. Krahmer, J. et al. Time-resolved interaction proteomics of the GIGANTEA protein under diurnal cycles in Arabidopsis. FEBS Lett. 593, 319-338 (2019).

26. Ewan, R. et al. Deubiquitinating enzymes AtUBP12 and AtUBP13 and their tobacco homologue NtUBP12 are negative regulators of plant immunity. New Phytol. 191, 92-106 (2011).

27. Derkacheva, M. et al. H2A deubiquitinases UBP12/13 are part of the Arabidopsis polycomb group protein system. Nat. Plants 2, 16126 (2016).

28. Jeong, J. S., Jung, C., Seo, J. S., Ki, J. K. \& Chua, N. H. The deubiquitinating enzymes UBP12 and UBP13 positively regulate MYC2 levels in jasmonate responses. Plant Cell 29, 1406-1424 (2017).

29. Zhou, H., Zhao, J., Cai, J. \& Patil, S. B. UBIQUITIN-SPECIFIC PROTEASES function in plant development and stress responses. Plant Mol. Biol. 94, 565-576 (2017).

30. An, Z. et al. Regulation of the stability of RGF1 receptor by the ubiquitinspecific proteases UBP12/UBP13 is critical for root meristem maintenance. Proc. Natl Acad. Sci. USA 115, 1123-1128 (2018).

31. Kim, Y. et al. ELF4 regulates GIGANTEA chromatin access through subnuclear sequestration. Cell Rep. 3, 671-677 (2013).

32. Takase, T. et al. LOV KELCH PRTEIN2 and ZEITLUPE repress Arabidopsis photoperiodic flowering under non-inductive conditions, dependent on FLAVIN-BINDING KELCH REPEAT F-BOX1. Plant J.: Cell Mol. Biol. 67, 608-621 (2011)

33. Zielinski, T., Moore, A. M., Troup, E., Halliday, K. J. \& Millar, A. J. Strengths and limitations of period estimation methods for circadian data. PLOS ONE $\mathbf{9}$, e96462 (2014).

34. Komander, D., Clague, M. J. \& Urbe, S. Breaking the chains: structure and function of the deubiquitinases. Nat. Rev. Mol. Cell Biol. 10, 550-563 (2009).

35. Mevissen, T. E. T. \& Komander, D. Mechanisms of deubiquitinase specificity and regulation. Annu. Rev. Biochem. 86, 159-192 (2017).

36. Pudasaini, A. \& Zoltowski, B. D. Zeitlupe senses blue-light fluence to mediate circadian timing in Arabidopsis thaliana. Biochemistry 52, 7150-7158 (2013).

37. Scoma, H. D. et al. The de-ubiquitinylating enzyme, USP2, is associated with the circadian clockwork and regulates its sensitivity to light. PLOS ONE $\mathbf{6}$, e25382 (2011)

38. Luo, W. et al. CLOCK deubiquitylation by USP8 inhibits CLK/CYC transcription in Drosophila. Genes Dev. 26, 2536-2549 (2012). 
39. Yang, Y. et al. Regulation of behavioral circadian rhythms and clock protein PER1 by the deubiquitinating enzyme USP2. Biol. Open 1, 789-801 (2012).

40. Hirano, A. et al. USP7 and TDP-43: pleiotropic regulation of cryptochrome protein stability paces the oscillation of the mammalian circadian clock. PLoS ONE 11, e0154263 (2016).

41. Papp, S. J. et al. DNA damage shifts circadian clock time via Hausp-dependent Cry1 stabilization. Elife 4, https://doi.org/10.7554/eLife.04883 (2015).

42. Hirono, Y. \& Redei, G. P. Induced premeiotic exchange of linked markers in the angiosperm Arabidopsis. Genetics 51, 519-526 (1965).

43. Koornneef, M., Hanhart, C. J. \& van der Veen, J. H. A genetic and physiological analysis of late flowering mutants in Arabidopsis thaliana. Mol. Gen. Genet. 229, 57-66 (1991).

44. Salome, P. A. \& McClung, C. R. PSEUDO-RESPONSE REGULATOR 7 and 9 are partially redundant genes essential for the temperature responsiveness of the Arabidopsis circadian clock. Plant Cell 17, 791-803 (2005).

45. David, K. M., Armbruster, U., Tama, N. \& Putterill, J. Arabidopsis GIGANTEA protein is post-transcriptionally regulated by light and dark. FEBS Lett. 580, 1193-1197 (2006).

46. Lee, C. M. et al. Decoys untangle complicated redundancy and reveal targets of circadian Clock F-box proteins. Plant Physiol. 177, 1170-1186 (2018).

47. Clough, S. J. \& Bent, A. F. Floral dip: a simplified method for Agrobacteriummediated transformation of Arabidopsis thaliana. Plant J.: Cell Mol. Biol. 16, 735-743 (1998).

48. Pruneda-Paz, J. L., Breton, G., Para, A. \& Kay, S. A. A functional genomics approach reveals CHE as a component of the Arabidopsis circadian clock. Science 323, 1481-1485 (2009).

49. Nakamichi, N. et al. PSEUDO-RESPONSE REGULATORS 9, 7, and 5 are transcriptional repressors in the Arabidopsis circadian clock. Plant Cell 22, 594-605 (2010).

50. Lu, Q. et al. Arabidopsis homolog of the yeast TREX-2 mRNA export complex: components and anchoring nucleoporin. Plant J.: Cell Mol. Biol. 61, 259-270 (2010).

51. Wu, S. et al. A plausible mechanism, based upon short-root movement, for regulating the number of cortex cell layers in roots. Proc. Natl Acad. Sci. USA 111, 16184-16189 (2014).

52. Wu, F. H. et al. Tape-Arabidopsis sandwich -a simpler Arabidopsis protoplast isolation method. Plant Methods 5, 16 (2009).

53. Penfield, L. et al. Dynein-pulling forces counteract lamin-mediated nuclear stability during nuclear envelope repair. Mol. Biol. Cell, https://doi.org/ 10.1091/mbc.E17-06-0374 (2018).

54. Schindelin, J. et al. Fiji: an open-source platform for biological-image analysis. Nat. Methods 9, 676-682 (2012)

55. Perkins, D. N., Pappin, D. J., Creasy, D. M. \& Cottrell, J. S. Probability-based protein identification by searching sequence databases using mass spectrometry data. Electrophoresis 20, 3551-3567 (1999).

56. Teo, G. et al. SAINTexpress: improvements and additional features in significance analysis of INTeractome software. J. Proteom. 100, 37-43 (2014).

57. Goldfarb, D., Hast, B. E., Wang, W. \& Major, M. B. Spotlite: web application and augmented algorithms for predicting co-complexed proteins from affinity purification-mass spectrometry data. J. Proteome Res. 13, 5944-5955 (2014).

58. Bleckmann, A., Weidtkamp-Peters, S., Seidel, C. A. \& Simon, R. Stem cell signaling in Arabidopsis requires CRN to localize CLV2 to the plasma membrane. Plant Physiol. 152, 166-176 (2010).

59. Rast, M. I. \& Simon, R. Arabidopsis JAGGED LATERAL ORGANS acts with ASYMMETRIC LEAVES2 to coordinate KNOX and PIN expression in shoot and root meristems. Plant Cell 24, 2917-2933 (2012).

60. Earley, K. W. et al. Gateway-compatible vectors for plant functional genomics and proteomics. Plant J. 45, 616-629 (2006).
61. Lee, C. M., Adamchek, C., Feke, A., Nusinow, D. A. \& Gendron, J. M. Mapping protein-protein interactions using affinity purification and mass spectrometry. Methods Mol. Biol. (Clifton, N. J.) 1610, 231-249 (2017).

62. Vizcaino, J. A. et al. The PRoteomics IDEntifications (PRIDE) database and associated tools: status in 2013. Nucl. Acids Res. 41, D1063-D1069 (2013).

\section{Acknowledgements}

We thank Dr. David E. Somers for providing the anti-ZTL antibody. We would like to thank Dr. Nicole Clay and Dr. Jimi Miller for kindly sharing pABind vectors and the assistance with transient expression in the Nicotiana benthamiana experiments, the Keck Proteomics Facility at Yale for processing samples and analyzing proteomic data, Dr. Shirin Bahmanyar, Dr. Marshall Delise, and Dr. Joseph Wolenski for assistance with confocal microscopy experiments. We would also like to thank Suyuna Eng Ren, Chris Adamchek, Catherine Chamberlin, Chris Bolick, Christine Ventura, Denise George, and Sandra Pariseau for technical and administrative support. We would like to thank Dr. Vivian Irish, Dr. Mark Hochstrasser, and Dr. Eric Bennet for their helpful comments and insight. This work was supported by NSF EAGER grant 1548538 (J.M.G.), NIH R35GM128670 (J.M.G.), Rudolph J. Anderson Fund Fellowship (C.M.L.), Forest B.H. and Elizabeth D.W. Brown Fund Fellowship (C.M.L. and W.L.), NIH GM007499 (A.F.), The Gruber Foundation (A.F.), and NSF GRFP DGE-1122492 (A.F.).

\section{Author contributions}

J.M.G. and C.M.L. conceived of the project. C.M.L., M.W.L., A.F., W.L. and A.M.S. conducted the experiments and analyzed the data. J.M.G., A.F. and C.M.L. wrote the manuscript.

\section{Additional information}

Supplementary Information accompanies this paper at https://doi.org/10.1038/s41467019-11769-7.

Competing interests: The authors declare no competing interests.

Reprints and permission information is available online at http://npg.nature.com/ reprintsandpermissions/

Peer review information: Nature Communications thanks Antony Dodd and other anonymous reviewer(s) for their contribution to the peer review of this work. Peer reviewer reports are available.

Publisher's note: Springer Nature remains neutral with regard to jurisdictional claims in published maps and institutional affiliations.

Open Access This article is licensed under a Creative Commons Attribution 4.0 International License, which permits use, sharing, adaptation, distribution and reproduction in any medium or format, as long as you give appropriate credit to the original author(s) and the source, provide a link to the Creative Commons license, and indicate if changes were made. The images or other third party material in this article are included in the article's Creative Commons license, unless indicated otherwise in a credit line to the material. If material is not included in the article's Creative Commons license and your intended use is not permitted by statutory regulation or exceeds the permitted use, you will need to obtain permission directly from the copyright holder. To view a copy of this license, visit http://creativecommons.org/ licenses/by/4.0/.

(C) The Author(s) 2019 